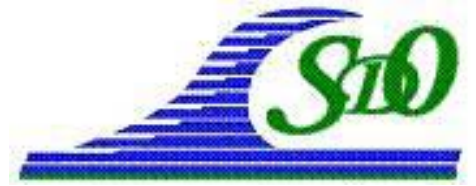

\title{
Etude préliminaire des mascarets d'Aquitaine
}

\section{Jean-Paul PARISOT ${ }^{1}$, Philippe BONNETON ${ }^{1}$, Natalie BONNETON ${ }^{1}$, Julien VAN-DE-LOOCK ${ }^{1}$, Bruno CASTELLE ${ }^{1}$, Vincent MARIEU ${ }^{1}$, Aldo SOTTOLICHIO ${ }^{\mathbf{1}}$, Guillaume DETANDT ${ }^{\mathbf{1}}$, Georges OGGIAN ${ }^{\mathbf{1}}$, Herve DERRIENNIC ${ }^{1}$, Stéphane BUJAN ${ }^{1}$, Marion TISSIER ${ }^{1}$, Patrice CASTAING ${ }^{1}$, Pierre LUBIN ${ }^{2}$, Hubert CHANSON ${ }^{3}$, Fabrice PRADALIER ${ }^{1}$, Roger MARCEL ${ }^{1}$}

1. Université Bordeaux 1. OASU/EPOC UMR 5805. Avenue des facultés, 33405 Talence, France.

parisot@epoc.u-bordeauxl.fr ; p.bonneton@epoc.u-bordeauxl.fr ; n.bonneton@epoc.u-bordeauxl.fr; Julien.Van-De-Loock@ensem.inpl-nancy.fr; b.castelle@epoc.u-bordeaux1.fr;v.marieu@epoc.u-bordeaux1.fr ; a.sottolichio@epoc.u-bordeauxl.fr;g.detandt@epoc.u-bordeauxl.fr ; g.oggian@epoc.u-bordeauxl.fr; h.derriennic@epoc.u-bordeauxl.fr ; s.bujan@epoc.u-bordeauxl.fr;m.tissier@epoc.u-bordeauxl.fr ; p.castaing@epoc.u-bordeauxl.fr ; fabrice.pradalier@wanadoo.fr ; rmarcel@centres-animation.asso.fr

2. Université Bordeaux 1. Laboratoire TREFLE-ENSCBP. 16 avenue Pey-Berland, 33607 Pessac Cedex. France.lubin@enscpb.fr

3. School of Civil Engineering, The University of Queensland, Brisbane QLD 4072, Australie.h.chanson@uq.edu.au

\section{Résumé :}

En 2009, un groupe de chercheurs de l'Université Bordeaux 1 a mis en place un programme d'étude des mascarets d'Aquitaine (Gironde, Garonne et Dordogne) avec le soutien financier de la région d'Aquitaine et de l'Université de Bordeaux 1. Ce programme regroupe des compétences indispensables: une longue expérience en mesures intensives sur le terrain développées dans le laboratoire EPOC ; un savoir-faire en modélisation qui place les équipes bordelaises, en très bonne place au niveau international ; une connaissance de l'estuaire très large : biologie, chimie et transport sédimentaire (EPOC)... Les temps forts de ce projet sont 2 campagnes de mesures, une campagne exploratoire en cours, et une campagne intensive programmée à l'automne 2010 pour une étude hydrodynamique de gros mascarets. Ce document présente les résultats préliminaires de cette étude, ainsi que les projets pour la campagne à venir, avec un survol de nos connaissances fragmentaires sur les mascarets aquitains, qui remontent à plus de 15 siècles.

Mots-clés :

Mascaret - Marées - Turbidité - Gironde - Dordogne - Garonne 


\section{Introduction}

Le mascaret, phénomène fortement médiatisé en particulier en Aquitaine (figure 1) et fortement pratiqué par les surfeurs depuis près de 40 ans, est peu étudié par la communauté scientifique en raison de sa complexité. En effet, il s'agit de la combinaison de phénomènes qui déjà individuellement sont des problèmes difficiles : propagation de la marée en milieu peu profond, genèse d'un ressaut hydraulique, hydrologie des fleuves, morphologie du fond.

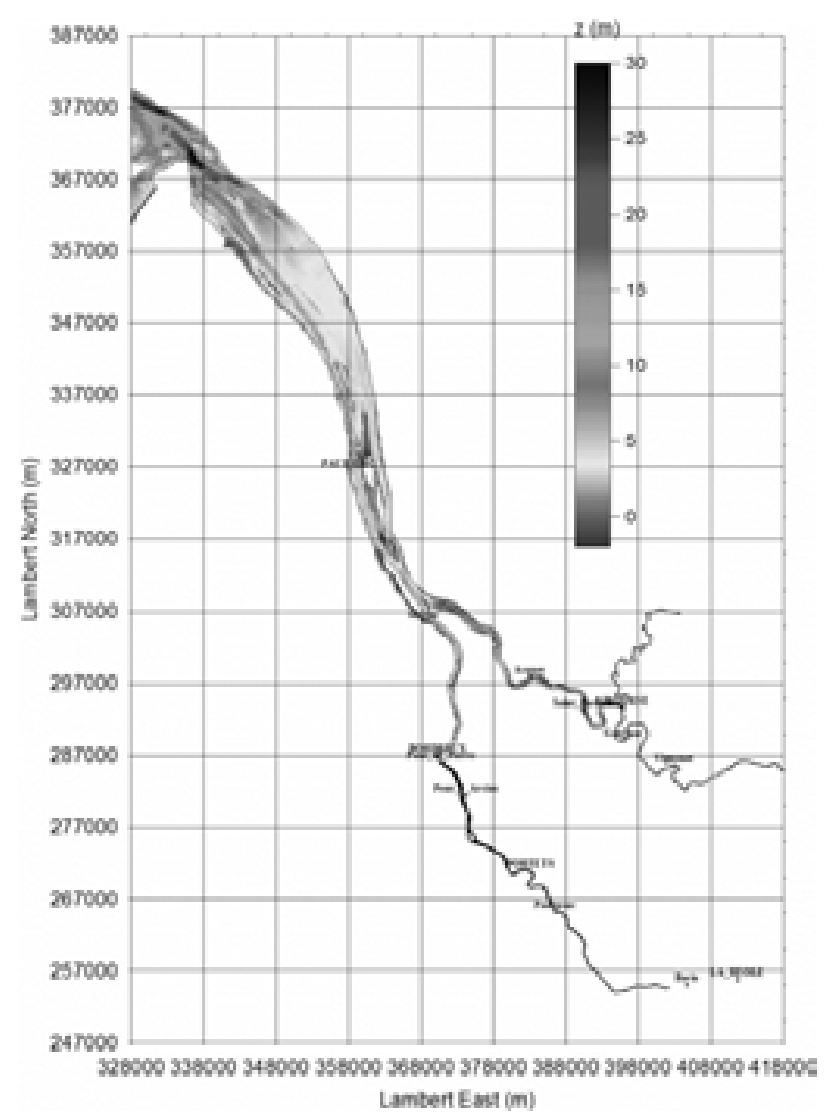

Figure 1. Sur le système fluvio-estuarien Gironde, Dordogne et Garonne (GGD), le mascaret se forme depuis Macau (grande ile située à proximité du Bec d'Ambès, confluent de la Garonne et de la Dordogne) et a été signalé jusqu'à Barie sur la

Garonne et Vignonnet sur la Dordogne soit à plus de $150 \mathrm{~km}$ de l'entrée de l'estuaire (Le Verdon). La zone d'expérimentation se trouve sur la commune de Podensac.

Le mascaret est une onde de translation appelée aussi ressaut en translation qui se forme dans certains estuaires lorsque les conditions de marée et de débit sont favorables. Le mascaret se propage vers l'amont à la renverse de la marée, juste après l'étale de marée basse. Ce phénomène peut être observé dans plusieurs estuaires de par le monde. Les figures 2 et 3 montrent deux photographies des mascarets de la Dordogne et de la Garonne. Suivant la forme de l'estuaire et les conditions hydrodynamiques on peut 
observer deux types de mascaret : le mascaret déferlant et le mascaret ondulé. C'est ce dernier qu'on observe dans le système GGD (Gironde, Garonne et Dordogne), ainsi que dans la plupart des estuaires à mascarets. Ce dernier a alors une forme ondulante et le front du mascaret est suivi par un train d'ondulations appelées "éteules".

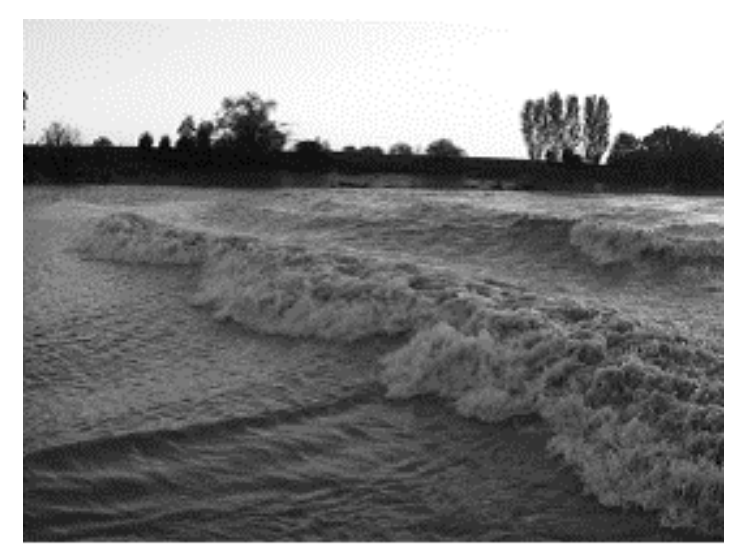

Figure 2. Garonne à Cambes le 21/09/2005.

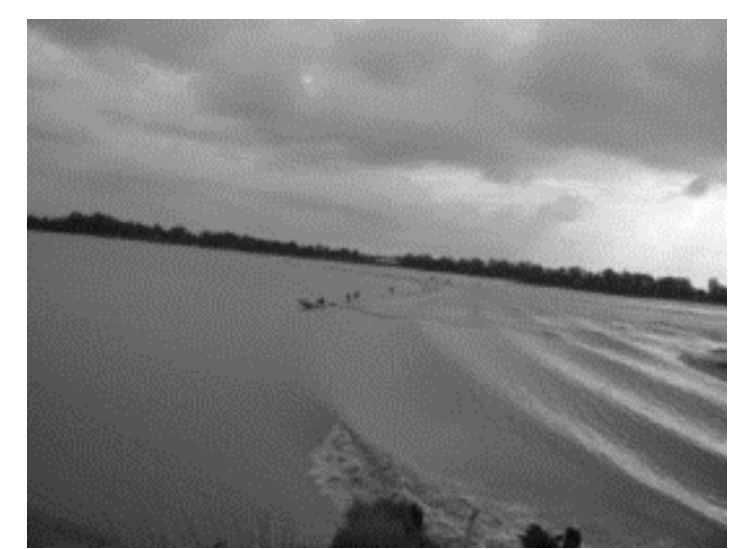

Figure 3. Dordogne à La Chapelle le 20/09/2008.

Si la modélisation physique et des études théoriques ont permis d'avancer dans la compréhension de ce phénomène (CHANSON, 2005), la dynamique ondulatoire et turbulente du mascaret reste mal connue, en particulier de par la difficulté d'étudier en milieu naturel ce phénomène extrêmement variable. Or le mascaret a un fort impact sur l'hydrodynamique sédimentaire et plus généralement l'écologie du système GGD. Les mouvements turbulents intenses associés au mascaret provoquent l'affouillement des sédiments, la mise en suspension des sédiments fins et leur transport vers l'amont. De par son impact sur le fond sédimentaire ainsi que par l'entrânement d'air dans le déferlement, le mascaret joue un rôle important dans les processus géochimiques. Plusieurs études menées dans d'autres environnements ont démontré l'impact du mascaret sur le transport de sédiment (TESSIER \& TERWINDT, 1994 ; CHEN et al., 1990 ; BARTSCH-WINKLER \& LYNCH, 1988). Ces études ont mis en évidence que le passage du mascaret provoque un affouillement du fond vaseux, une mise en suspension des matériaux fins, et l'advection de ces matériaux vers l'amont. Dans les fleuves du système GGD, ces phénomènes n'ont toujours pas été mesurés finement, et donc l'impact relatif du mascaret sur la stabilité des berges, sur l'érosion (ou la sédimentation) dans le chenal et sur les flux sédimentaires est mal connu. Il est donc crucial d'évaluer son effet relatif par rapport aux courants de marée, en vue d'en tenir compte dans des futures modélisations du transport sédimentaire. Par ailleurs, le lien entre la typologie de l'onde et les effets respectifs sur la remise en suspension n'est toujours pas clairement établi. En effet, d'après DONNELLY et CHANSON (2005) et KOCH et CHANSON (2008), les mascarets ondulés induisent le transport particulaire 
le plus intense (par maintien en suspension plus important), cependant WOLANSKI et al. (2004) estiment que le mascaret déferlant engendre des flux d'érosion bien plus importants que le mascaret ondulé, et possède donc un impact prépondérant sur le transport. L'absence presque complète de mesures in situ sur les mascarets du monde s'explique par la difficulté d'expérimentation dans un milieu que l'on pourrait qualifier d'hostile (milieu très agité et turbulent; accès difficile des zones à mascarets...). La simulation numérique aboutit actuellement à un stade où la modélisation d'un mascaret est possible, ceci grâce aux gros efforts qui ont été réalisés depuis quelques années dans le domaine de l'étude des écoulements à surface libre (LUBIN et al., 2010 ; CIENFUEGOS et al., 2010 ; BONNETON et al., submitted)

\section{Que savons-nous des mascarets d'Aquitaine}

Actuellement, il y a plus de 80 sites à mascarets dans le monde (BARTSCH-WINKLER \& LYNCH, 1988), avec de nombreuses occurrences sur les côtes européennes et dans l'estuaire de l'Amazone. L'Europe est effectivement riche en mascarets avec plus de 15 mascarets en Angleterre (Severn, Trent, etc.), Aquitaine (GGD) et en Normandie (Sée, Sélune, Couesnon, etc.). Bien que ce phénomène possède un nom gascon (tableau 1), il n'y a pratiquement pas eu d'études sur les mascarets d'Aquitaine.

Tableau 1. Définition du mot mascaret d'après le Petit Robert.

MASCARET. (XVIe; mot gascon «boeuf tacheté », de mascara « machurer, tacheter », par métaphore, d'un animal bondissant). Longue vague déferlante produite dans certains estuaires par la rencontre du flux et du reflux. Le mascaret de la Gironde, de la Seine.

En effet, la plus ancienne référence connue est tirée de l'ouvrage de PALISSY (1586), né à Agen en 1510, qui propose une théorie (Tableau 2), d'après laquelle le mascaret est produit par des masses d'air emprisonnées dans le sol et qui ressortent en faisant gonfler le niveau de l'eau.

Tableau 2. Extrait de l'ouvrage de PALISSY (1586) où il présente sa théorie (qui paraît aujourd'hui très fantaisiste).

«L'on appelle mascaret une grande montaigne d'eau qui se fait en la rivière de Dourdongne, vers les contrées de Libourne, et ladite montagne ne se fait sinon au temps d'esté: mesmes ès saisons les plus paisibles, et lorsque les eaux sont les plus tranquilles, et tout en un moment, en une saison inconneue, la montagne d'eau se forme en un instant et fait une course, quelquefois bien longue, le long de l'eau, et quelquefois plus courte: et lorsque la montaigne fait son cours, elle renverse tous les bateaux qu'elle trouve en son chemin: parquoy les habitans limitrophes de la rivière, quand ils voyent le mascaret en sa formation, ils se prennent soudain à crier de toutes parts: garde le mascaret, garde le mascaret, et les bateliers qui pour lors sont en la rivière s'enfuyent ès rivages, pour sauver leurs vies, qui autrement seroyent près de leur fin» 


\title{
XI $I^{\text {èes }}$ Journées Nationales Génie Côtier - Génie Civil Les Sables d'Olonne, 22-25 juin 2010
}

Tableau 3. Extrait de la mention historique du mascaret de la Dordogne au IVe siècle de notre ère.

\begin{abstract}
Il est un lieu où insensiblement vous confondez vos cours déjà ralentis, Garonne, dont la source coule d'un rocher riche en eau, et toi, Dordogne moussue qui te hâtes vers la plaine par une pente aussi rapide pour y couler ensuite sur le sable de ton lit. La mer, à cet endroit, court contre le courant et par le mouvement constant de flux et de reflux repousse ou invite les ondes qui roulent les rivières. Mais lorsque la Garonne, refoulée par les phases croissantes de la lune, rassemble de son côté ses flots en forme de barre, elle rétrograde précipitamment en un cours impétueux et semble alors retourner vers sa source non par un mouvement de reflux mais par une pente naturelle.

Il dirige ses pas aux lieux où l'Océan, poussé par la marée, fait refluer la Garonne et la répand à travers champs : la mer envahit le fleuve; le flot amer escalade les eaux douces et, jetée avec force dans le lit de la rivière, l'onde salée vogue sur des profondeurs qui lui sont étrangères.

Traduction Stephanie Dupays
\end{abstract}

La plus ancienne description du phénomène serait due à Sidoine Apollinaire (Caius Sollius Apollinaris Sidonius, 430-486), évêque de Clermont-Ferrand. Dans une longue description de son voyage en Aquitaine (Tableau 3), il décrit le phénomène de la marée et du mascaret au niveau du bec d'Ambès; le tableau 2 contient le texte en latin et sa traduction. On peut noter que ces 2 références anciennes ne concernent pas la Garonne mais seulement la Dordogne. Une explication simple, serait l'absence de mascaret sur la Garonne, qui de toute façon ne remontait pas en amont de Bordeaux à la fin du $19^{\mathrm{e}}$ siècle (COMOY, 1881). L'autre explication provient du fait que ce phénomène s'appelait autrement sur la Garonne puisque les textes anciens l'appellent le montant (COULON, 1864). La première explication moderne est réalisée par BREMONTIER (1809) ingénieur des Ponts et Chaussées, qui entre 1770 et 1780, a réalisé en Aquitaine de nombreuses études concernant les ports et l'aménagement des dunes. Dans son ouvrage, il explique que le mascaret est produit par le déferlement de l'onde marée (tableau 4).

Il y aura par la suite une littérature abondante, en particulier sur le mascaret de la Seine qui a donné lieu à de nombreuses publications jusqu'à sa disparition en 1963 (MALANDAIN, 1988). Avant les travaux d'endiguement (digue du Ratier), le mascaret atteignait une hauteur de $2.50 \mathrm{~m}$ à Caudebec-en-Caux, là où maintenant il a disparu. Mais il existe encore au débarcadère du bac de Sahurs en aval de Rouen pour les coefficients supérieurs à 100. La thèse de COMOY (1881) décrit avec beaucoup de détails, le mascaret de la Seine ainsi que celui des autres fleuves de France où des mascarets se produisaient autrefois (Charente, Adour...). On y trouve par exemple un profil de la Garonne et une description de son mascaret qui à l'époque ne remontait pas au-delà de Portets (130 km du Verdon) alors qu'aujourd'hui, il remonte plus de $15 \mathrm{~km}$ en amont. De plus, il présentait son maximum en aval de Bordeaux, dans des zones où il est inexistant actuellement, puisqu'il se développe principalement en amont de Bordeaux. 
Tableau 4. Extrait de l'ouvrage de N. Brémontier.

«Il est de fait, dit- il, que la marée dans la Gironde, depuis Royan, met trois heures pour se rendre dans les environs de Libourne et de Bordeaux, qu'elle a dû s'élever successivement pendant ce temps, par petites lames ou couches de 15 millimétres réduits d'épaisseur par minute, et par conséquent de 3 mètres ou 9 pieds sur les bords de la mer. Il est de fait encore que les courants du descendant, suivant qu'ils sont plus ou moins rapides, retardent plus ou moins la marche ou le développement de cette première petite lame; que la deuxième qui la suit ayant moins de difficultés à vaincre, doit acquérir plus de vitesse et parcourir en moins de temps l'espace déjà parcouru par la première, avec laquelle elle se trouvera nécessairement bientôt réunie; que, par la même raison, chacune des lames qui suivent acquérant un degré de vitesse de plus que celles qui les précèdent, elles • finissent toutes par se réunir au même point que toutes ces petites lames une fois réunies, abstraction faite de diverses qui peuvent en déranger, en atténuer la marche ou l'accélérer, doivent nécessairement former une grosse lame et c'est cette grosse à laquelle on a donné les divers noms de flot, de première vague, de barre et de mascaret.»

\section{Synthèse des données existantes sur les mascarets aquitains}

L'inventaire des études scientifiques disponibles est rapide, car il n'existe que deux études concernant les mascarets aquitains.

\subsection{Travaux de DESTRIAU (1951)}

A partir d'observations et de mesures photographiques (hauteur, vitesse) s'étendant sur les années 1950 et 1951, les mascarets de la Garonne et surtout celui de la Dordogne sont décrits, avec une carte détaillée des zones à mascarets sur la Dordogne En particulier, il tente d'expliquer un phénomène bien connu qui tend à amplifier le mascaret 1 à 2 jours après le plus gros coefficient de marée.

\subsection{Travaux de NAVARRE (1995)}

Des mesures ont été effectuées à Saint-Pardon sur la Dordogne les 25 et 26 avril 1990. Deux courantomètres mesurant des vitesses horizontales et verticales ont été installés en surface et sur le fond. Une échelle de marée a été filmée afin de relever l'évolution de la hauteur d'eau et un sondeur embarqué sur le bateau placé dans le lit de la Dordogne a également mesuré les variations de hauteur. La renverse brutale s'accompagne de 2 familles d'ondulations, la première d'un période de $2.6 \mathrm{~s}$ et la seconde de $80 \mathrm{~s}$. Si les ondulations à haute fréquence sont facilement observables, la grande oscillation d'une longueur d'onde d'environ 300 m, n'a jamais été décrite.

\section{Le projet MASCARET2010}

Une grande campagne d'observations a eu lieu le 20 septembre 2009, ce qui a permis de caractériser complètement un gros mascaret (coefficient 110). Cette campagne a consisté à déployer environ 50 personnes et un bateau sur tout le parcours du mascaret, 
soit $2 \times 100 \mathrm{~km}$. De plus le mascaret a été filmé avec 2 caméras, sur tout son développement.

De nombreuses études préliminaires ont permis de converger vers un site d'étude situé dans la zone Podensac/Rions (PK 40 à environ $140 \mathrm{~km} \mathrm{du} \mathrm{Verdon)} \mathrm{où} \mathrm{le} \mathrm{mascaret} \mathrm{est}$ bien formé sur toute la largeur, déferlant en aval. Quatre capteurs (Acoustic Doppler Courrent Profiler, ADCP, Acoustic Doppler Velocimeter, ADV et deux capteurs de pression P1 et P2) ont été mis en place, ainsi qu'une base GPS implantée afin d'avoir une référence pour les GPS cinématiques pour les mesures de positionnement. L'ADCP et l'ADV ont été installés dans des structures amagnétiques (INOX 316L). Ces structures, ont dû être étudiées préalablement pour accueillir et protéger au mieux les instruments. En effet, le milieu est hostile, en particulier au printemps où la Garonne charrie de nombreux troncs d'arbres. Les 2 structures en forme de pyramide et d'igloo sont lestées avec environ $50 \mathrm{~kg}$ de plomb et assurées avec des corps-morts de $450 \mathrm{~kg}$ (déposés avec le soutien technique de la station VNF de Cadillac). Un mat de $12 \mathrm{~m}$ de hauteur supportant 2 caméras a également été testé. De plus durant la mission de septembre 2010, le mascaret sera suivi par un drone (http://www.fly-n-sense.com/) dont des essais ont été réalisés en septembre 2009.

Hormis un capteur de pression (qui a couvert un cycle de marées complet), les instruments ont été retirés le 5 mars. Un dépouillement rapide a montré qu'ils ont bien fonctionné, à part le capteur de pression de l'ADV. La figure 4 montre la variation de profondeur mesurée par le capteur de pression de l'ADCP. Le signal est très régulier avec un fort marnage, malgré la grande distance nous séparant de l'embouchure.
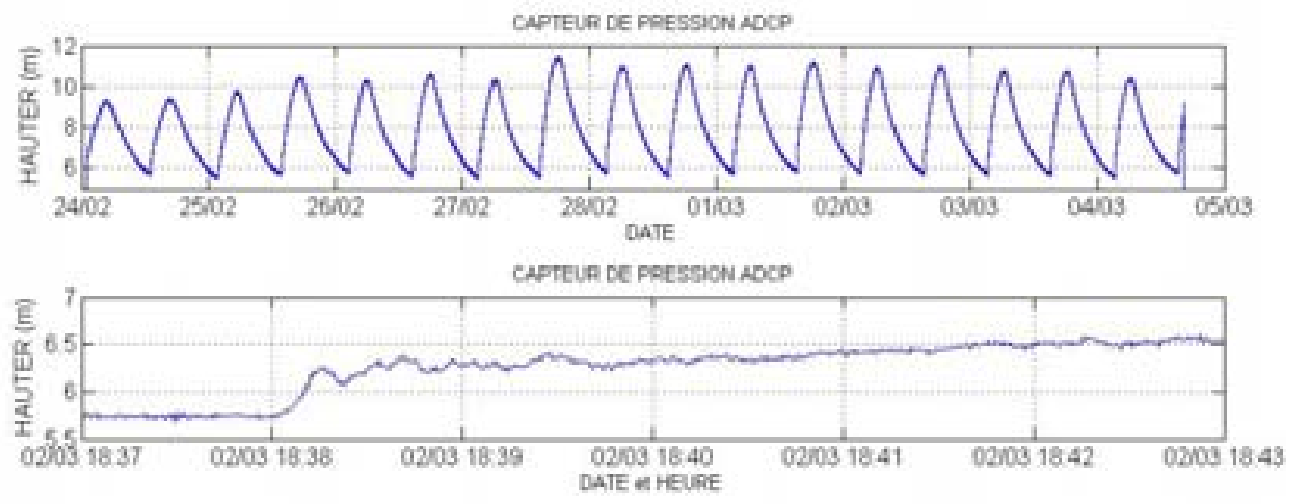

Figure 4. Relevé du capteur de pression de l'ADCP et zoom sur le passage du petit mascaret du 2 mars vers $18 \mathrm{~h} 38$.

L'acquisition a coïncidé avec la tempête Xynthia dont l'épisode de crue dû à une surcote de plus d'un mètre est bien visible durant la nuit du 27/28 février. En cette période, en raison des débits importants, il est bien connu que de gros mascarets ne se forment pas. Quand on examine les variations de hauteur d'eau, juste après la basse mer, on constate 
avec surprise de petits mascarets de faible amplitude, d'environ $50 \mathrm{~cm}$ qui apparaissent à partir du 26 février au soir soit avec des coefficients de 76. La présence de ce signal de mascaret est extrêmement prometteuse car cela confirme que la zone sélectionnée est favorable à une campagne de mesures.

\section{Conclusion}

Les gros efforts déployés dans cette campagne expérimentale, mise en place pour tester des méthodes et des moyens, ont porté leurs fruits en raison des résultats prometteurs obtenus. En effet, dans des conditions très défavorables (débits importants, grande profondeur choisie pour des raisons de sécurité...) les mascarets sont présents avec un ressaut de l'ordre de $50 \mathrm{~cm}$ et des coefficients inférieurs à 80. D'autre part, les structures supportant les instruments se sont bien comportées dans des conditions hostiles laissant présager une campagne fructueuse en septembre 2010.

\section{Références bibliographiques}

BARTSCH-WINKLER S., LYNCH B. (1988). Catalogue of Worldwide Tidal Bore Occurrences and Characteristics. US Geological Survey Circular 1022

BONNETON P., BARTHELEMY E., CARTER J.D., CHAZEL F., CIENFUEGOS E., LANNES D., MARCHE F., TISSIER M. (submitted). Fully nonlinear weakly dispersive modelling of wave transformation, breaking and runup. European Journal of Mechanics - B/Fluids.

BREMONTIER N. (1809). Recherches sur le mouvement des ondes. Firmin Didot, Paris.

CHANSON H. (2005). Mascaret, Aegir, Pororoca, Tidal Bore. Quid ? Où? Quand? Comment? Pourquoi ? La Houille Blanche, ${ }^{\circ}$ 3, pp 103-114. doi:10.1051/lhb:200503014

CHEN J., LIU C., ZHANG C., WALKER H.-J. (1990). Geomorphological Development and Sedimentation in Qiantang Estuary and Hangzhou Bay. J. of Coastal Res., Vol. 6, n 3, pp 559-572.

CIENFUEGOS R., BARTHELEMY E., BONNETON P. (2010). A wave-breaking model for Boussinesq-type equations including mass-induced effects. J. Waterway, Port Coastal and Ocean Engrg, 136, pp 10-26. doi:10.1061/(ASCE)WW.1943-5460.0000022

COMOY M. (1881). Etude pratique sur les marées fluviales et notamment sur le mascaret. Gauthier-Villards, Paris, 399 p.

COULON L. (1864). Les rivières de France, ou Description géographique et historique du cours et débordement des fleuves, rivières, fontaines, lacs et estangs qui arrousent les provinces du royaume. G. Clousier. Paris.

DESTRIAU G. (1951). Le mascaret. Annales des Ponts et Chausses, $\mathrm{n}^{\circ}$ 121, pp 609-635. 


\section{XI $I^{\text {èes }}$ Journées Nationales Génie Côtier - Génie Civil \\ Les Sables d'Olonne, 22-25 juin 2010}

DONNELLY C., CHANSON H. (2005). Environmental Impact of Undular Tidal Bores in Tropical Rivers. Environmental Fluid Mechanics, Vol. 5, ${ }^{\circ}$ 5, pp 481-494. doi:10.1007/s10652-005-0711-0

KOCH C., CHANSON H. (2008). Turbulent Mixing beneath an Undular Bore Front. Journal of Coastal Research, Vol. 24, n 4, pp 999-1007. doi:10.2112/06-0688.1

LUBIN P., GLOCKNER S., CHANSON H. (2010). Numerical Simulation of a Weak Breaking Tidal Bore. Mechanics Research Communications, Vol. 37, n 1, pp 119-121 doi:10.1016/j.mechrescom.2009.09.008

MALANDAIN J.J. (1988). La Seine au Temps du Mascaret. Le Chasse-Marée, ${ }^{\circ} 34$, pp 30-45

NAVARRE P. (1995). Aspects physiques du caractère ondulatoire du mascaret en Dordogne. DES. Université Bordeaux 1.

PALISSY B. (1586). Discours Admirable de la Nature des Eaux et Fontaines tant Naturelles qu'Artificielles. Martin Le Jeune. Paris.

TESSIER B., TERWINDT H.J. (1994). An example of soft sediment deformations in an intertidal environment: the effect of a tidal bore. Comptes Rendus Académie des Sciences, Serie II, 319, pp 217-223.

WOLANSKI E., WILLIAMS D., SPAGNOL S., CHANSON H. (2004). Undular tidal bore dynamics in the Daly Estuary, Northern Australia. Estuarine, Coastal and Shelf Science, 60, pp 629-636. doi:10.1016/j.ecss.2004.03.001 
Thème 3 - Instrumentation, mesures, imagerie et télédétection 